\title{
Utilizar el cálculo en la escuela: la pro- gramación de una situación significativa
}

\section{Eulàlia Bassedas}

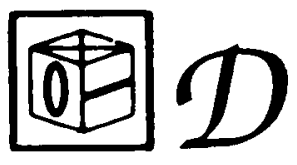

La necesidad de que los conceptos y signos matemáticos estén ligados desde sus inicios a actividades familiares $y$ significativas es la idea que guía esta experiencia didáctica, en que la construcción de los conocimientos matemáticos en niños de 4 a 8 años se realiza en talleres con juegos habituales como la oca, el parchís o los naipes.

\section{CONTEXTUALIZACION DE LA EXPERIENCIA}

El trabajo que presentamos es el resultado de una tarea compartida por diversos profesionales dedicados a la educación. Podría decirse que se trata de una experiencia en la que conjuntamente algunos profesionales hemos buscado diferentes soluciones a los problemas que se planteaban en la práctica educativa. En lo esencial, nuestro interés se centraba en buscar nuevos caminos que nos ayudaran a perfeccionar y a avanzar en un intento de hacer más significativo el aprendizaje en las diferentes áreas. En este caso, además, tratábamos de profundizar en la organización de clase mediante rincones o talleres de juego/trabajo, y concretamente en el área de matemáticas, en el aprendizaje de cálculo que se pretende realizar con los alumnos de 4 a 8 años en la escuela.

La experiencia se ha realizado en una escuela pública de una población del cinturón industrial de Barcelona, en la que ya había una tradición de innovación, en el sentido de que se habían iniciado algunas experiencias que habían representado para la escuela la necesidad de replantearse metodologías, objetivos de escuela, organización de los contenidos... (podemos citar en este sentido el planteamiento de la atención a la diversidad en los distintos ciclos, la introducción de métodos de aprendizaje del catalán desde las primeras edades, la introducción de talleres de pretecnología en ciclo medio,...)

Los inicios del trabajo que presentamos surgen en el curso 1986-87 mediante el replanteamiento por parte de las maestras de parvulario, de ciclo inicial y de apoyo para la educación especial, del modo de llevar a cabo el aprendizaje de las matemáticas, que consideraban poco motivador para los alumnos representando un aprendizaje poco funcional; al mismo tiempo había un interés en 
profundizar en la metodología de rincones/talleres, es decir, en intentar introducir los contenidos propios de las diferentes áreas curriculares, a partir de una organización de clase por talleres. Desde un principio, este trabajo se ha realizado conjuntamente entre las maestras de la escuela y la firmante del artículo, que era en aquel momento la psicóloga del Equipo de Asesoramiento Psicopedagógico que atendía la escuela. El trabajo en común se ha planteado siempre como una colaboración en la que cada una de las partes ha tenido algo diferente que aportar al grupo. Así pues, pensamos que trabajos de este tipo representan una investigación compartida por todos los que participan en ella, y en la que se trata de buscar soluciones a los problemas que la práctica educativa cotidiana presenta (Bassedas y otros, 1990).

\section{APRENDER ARITMETICA JUGANDO}

Las concepciones actuales sobre la enseñanza de las matemáticas ponen el énfasis en la utilidad que deben tener los conceptos y procedimientos adquiridos en la escuela para poder resolver problemas prácticos. Igualmente (M.E.C. 1989), en la escolaridad primaria, se propone atender básicamente los aspectos de habilidades de cálculo mental y que los alumnos sean capaces de hacer estimaciones del tipo de operaciones que será necesario realizar para resolver un determinado problema y de los resultados que se encontrarán. Asimismo, se incide en la importancia de fomentar una actitud positiva hacia la posibilidad de buscar soluciones a los problemas matemáticos con los que nos enfrentamos, así como buscar situaciones en las que los alumnos puedan entender qué son y para qué sirven las matemáticas.

Es decir, se hace un especial hincapié en que los aprendizajes matemáticos signifiquen para los niños de estas edades un instrumento intelectual más, que les permita identificar y resolver situaciones en un sentido amplio.

Otro de los aspectos a señalar en el planteamiento actual de la psicopedagogía de las matemáticas es la importancia otorgada al proceso de construcción individual de los conceptos matemáticos, proceso ligado a la resolución de situaciones-problema reales y concretas con los que se enfrenta individualmente $o$ en pequeño grupo.

En los ciclos que nos ocupan (educación infantil, y primer ciclo de primaria) es necesario incorporar ya estas ideas sobre lo que tiene que ser para los alumnos el aprendizaje de las matemáticas. Igualmente, nos interesa resaltar la importancia del modo que la escuela tenga de aproximar a los alumnos a esta área, para el posterior éxito en su aprendizaje. En este sentido, nos parece muy interesante otorgar un papel, en el trabajo con niños de estas edades, a los procedimientos informales (Baroody, 1988) que los alumnos hayan construido para resolver las situaciones matemáticas que inevitablemente se han encontrado antes de su entrada en la escuela. La matemática es un lenguaje aplicado a una realidad concreta, y lo que hace la escuela es ayudar a los alumnos a acceder a este lenguaje formal. Pero si entendemos que los niños ya tienen un pensamiento y un conocimiento matemático antes de la entrada a la escuela, será mucho más posible que seamos respetuosos con los conocimientos previos que aportan desde su experiencia extraescolar. Así, es imprescindible que los alumnos aprendan a manipular los signos propios del lenguaje formal, sólo a partir de la comprensión de su significado, hecho que es posible siempre que éstos se hayan utilizado en contextos familiares anteriormente. 
Las ideas expuestas sobre el aprendizaje de las matemáticas, nos llevan a señalar que entendemos que en la escuela hay multitud de situaciones en las que pueden aprenderse significativamente los conceptos y procedimientos propios de esta área y en las edades que nos ocupan. Sin embargo, es importante delimitar estas situaciones, analizar los conocimientos de los alumnos, e intervenir en consecuencia. En nuestra experiencia esto se ha realizado principalmente en los talleres de juego, pero hay que señalar que se utilizan también otras situaciones de vida cotidiana diarias o puntuales para aproximar a los alumnos al mundo de las matemáticas (votaciones, pasar lista, el calendario,...)

La situación de trabajo en talleres que se presenta a los alumnos está centrada en el juego mediante juegos de normas (la oca, el parchís, entre los más conocidos) y los juegos de naipes. Una vez realizado el análisis de estos juegos se ha podido concretar los contenidos de tipo matemático que los alumnos tienen que poner en marcha cuando están realizando una partida. Así, ha sido necesario hacer una selección del material de juego adecuado para cada una de las edades, y ofrecer a los alumnos el material correspondiente que permita ejercitar los conceptos matemáticos adecuados a los conocimientos previos que los alumnos tienen. En este sentido, las decisiones están entre presentar un tipo de juego u otro, o un material de juego con variaciones: un dado de cuatro, de seis, de diez o veinte caras, de cifras o con puntos, un tablero con numeración hasta el 10 o el 50 , etc.

Es importante señalar que consideramos que los alumnos, durante las sesiones de juegos de mesa, utilizan de un modo totalmente funcional los procedimientos que se espera que aprendan los alumnos de estas edades: contar, leer una cifra, descomponer en diferentes sumandos, aprenderse la serie numérica, hacer adiciones mentalmente, otorgar un ordinal a una serie, entre otros. Así pues, consideramos muy importante este aspecto que nos permite dejar de lado los aprendizajes mecánicos y no significativos de los procedimientos de cálculo.

\section{LA SITUACION DIDACTICA: EL FUNCIONAMIENTO DE LOS TALLERES}

La unidad didáctica que presentamos se basa en el trabajo por grupos en la clase. Hemos denominado talleres a la agrupación de alumnos que llevan a cabo una tarea en común y mediante la cual se pretende conseguir unos objetivos determinados previamente. Consideramos importante la interacción entre iguales en el sentido de que esto permite que los alumnos aprendan unos de otros, y que establezcan entre ellos los procesos de ayuda, comentario, ánimo que muchas veces la maestra no puede ofrecer a todos por un igual. Entendemos que mediante los procesos de interacción entre iguales se establecen lazos entre los diferentes niveles y capacidades de los alumnos que permiten avanzar a todos ellos en un sentido de mayor capacidad en el tema que se trabaja, en nuestro caso el cálculo.

A pesar del énfasis puesto en la importancia de la interacción entre los alumnos no podemos olvidar el papel que juega la maestra en estas situaciones de trabajo en pequeño grupo: su tarea se fundamenta en la observación de los procesos que realizan los alumnos en la resolución de las situaciones planificadas por ella y en la intervención adecuada que procure la ayuda educativa que el alumno requiere para avanzar en el proceso de construcción de los conceptos matemáticos. 
Los juegos están organizados por sesiones diferentes, siempre en situación de trabajo en pequeño grupo (máximo de 6 alumnos). A lo largo de la experiencia hemos visto que ha sido interesante organizar los talleres en base a 3 sesiones diferentes con cada juego.

Así, en la primera sesión, se hace una presentación del juego, sus normas y particularidades y seguidamente la maestra juega una partida con ellos.

En la segunda sesión, con el mismo juego, la maestra pasa a hacer un papel de observadora activa, tratando de hacer una observación de diversos aspectos que tienen que ver con los contenidos que se trabajan durante el juego: estrategias de recuento, dificultades o errores en la suma de los dados o cartas, tipo de interacción entre los alumnos del grupo. A partir de la observación de estos aspectos la maestra interviene o no en el sentido de permitir el avance del grupo o del alumno en particular. Para llevar a cabo este trabajo con el mayor conocimiento de los aspectos a observar, se han confeccionado unas pautas u hojas de observación que son de gran ayuda en este proceso. Más adelante se explicarán algunas de estas pautas en juegos concretos.

En la tercera sesión, que representa la última partida con el juego iniciado dos sesiones antes, se pasa, una vez finalizada, a rellenar en grupo, individualmente, con la ayuda de la maestra o sin ella (dependiendo del momento del juego, del curso y del grupo de alumnos) lo que hemos denominado la ficha-resumen. En ella se trata de hacer un recordatorio por escrito de algunos aspectos que se han realizado mediante la práctica durante el juego. Es en este momento cuando se inicia el paso al papel de algunos contenidos puestos en práctica durante el juego: escritura de la numeración, resolución de pequeños problemas planteados durante el juego mediante la adición o sustracción con los dados o cartas, descomposición de los números,... Es un momento en que pueden darse situaciones de discusión entre los alumnos en relación a los conceptos matemáticos trabajados hasta el momento en el juego.

A lo largo del curso, se repite semanalmente esta organización, hasta que todos los alumnos han realizado todos los juegos que se han programado en los talleres para aquel curso. Se acostumbra a realizar un promedio de diez juegos diferentes en cada uno de los niveles a lo largo del curso escolar. Las observaciones realizadas durante los años en los que se ha utilizado los juegos de este modo, nos permiten afirmar que durante un curso puede verse una gran variación en las estrategias de cálculo utilizados por los alumnos. Podemos asegurar que la ayuda aportada por la maestra - basada en una observación sistemática-, y por los compañeros es muy importante a la hora de favorecer el avance en la adquisición de los procedimientos de cálculo. En este sentido, podemos señalar que la observación de los procesos que realizan los alumnos es un instrumento básico en la evaluación formativa de la situación de aprendizaje en la que se encuentran.

\section{DESCRIPCION DE LOS JUEGOS}

Con el fin de ejemplificar estas tres sesiones descritas, pasaremos a explicar el modo cómo se plantean en dos juegos. En primer lugar, se explicará un juego de naipes tal como se utiliza en la clase de parvulario 5 años. A continuación, se comentará un juego de normas con dados utilizado en Segundo de E.G.B. 
a) El primer juego

El primer juego se denomina "Sietes» $^{1}$ y consiste en un juego de cartas en el que los niños tienen que formar el número 7 con una carta de las que se les ha repartido y todas las demás que están encima de la mesa y de las que otros se han descartado. Se trata pues de un juego en el que básicamente se trabaja la descomposición del número.

La observación en este juego se puede organizar en base a tres momentos:

1) antes del juego: modo de rapartir las cartas (igual número de cartas a cada niño/ña, reparte de uno en uno, de dos en dos, seguimiento de un orden en el reparto...).

2) durante el juego: lectura del número de las cartas (reconoce automáticamente el número y le otorga la cantidad correspondiente, tiene que reseguir con el dedo el número de objetos dibujados en la carta, cuenta los objetos con acciones visibles-golpes de cabeza, dedos,...); adición de los números para hacer 7 (cuenta el número de objetos de su carta y el de otras hasta conseguir llegar al 7, no necesita contar el número de su carta y busca contanto directamente en las otras cartas, busca directamente el número que le falta sin necesidad de contar con acciones visibles, se equivoca sistemáticamente en la adición de los números...).

3) después del juego: recuento del número de veces que ha completado 7; reconocimiento del ganador.

La ficha resumen realizada para este juego consta de los siguientes apartados (ver figura 1):

FIgURA 1

Nombre del alumno:

Fecha de juego:

Nombre del juego:

Número de niños que han jugado:

Número de veces que cada uno ha podido completar 7 :

Expresión gráfica de las diferentes combinaciones realizadas (de dos o de tres sumandos):

Nombre del compañero ganador:

\section{b) El segundo juego}

El segundo juego que presentamos lo denominamos «Obstáculos», y es un juego de fabricación casera, utilizado en $2 .^{\circ}$ de E.G.B. Se trata de una tablero de 112 casillas, en las que hay unos dibujos de vez en cuando que indican que los alumnos deben coger unas tarjetas con instrucciones. Hay dos tipos de instrucciones diferentes: la primera, en la que se trata de realizar acciones sobre el mismo tablero (avanzar, retroceder,...) y las otras en las que se trata de realizar una acción encomendada con un tiempo máximo de tres minutos. Si no se realiza la prueba, se retroceden cinco casillas. El material que se ha asignado a este juego, consta, además del tablero y las fichas correspondientes, de dos tipos de dados diferentes: uno de diez caras numerado del 0 al 9; y otro de seis 
caras con la consigna de multiplicar el número que ha salido en el dado de diez caras por 2 o por 3; es decir 3 caras en el que consta « $\times 2$ » y tres caras en el que consta « $x 3$ ». Se trata pues de un juego en el que básicamente se practica la adición, la multiplicación y el conocimiento de la numeración.

La observación puede realizarse también en relación a los tres momentos comentados anteriormente: antes, durante y después del juego.

a) antes del juego: repartición adecuada del número de fichas a cada jugador; cada niño coge las que le corresponde....

b) durante el juego: efectúa la operación de multiplicación automáticamente, con ayuda de los compañeros o de las tablas de la clase, se equivoca sistemáticamente y no se da cuenta, corrige,...; modo de contar las casillas que tiene que avanzar o retroceder: suma mental de la casilla a la que tendrá que desplazarse, cuenta casilla por casilla a partir de lo que le ha salido en los dados, suma con acciones visibles el resultado de los dados y la casilla en la que está antes de desplazar su ficha; lectura de las consignas de las tarjetas con instrucciones: lee y entiende, se apoya en sus compañeros para la comprensión y/o la lectura, ...

c) después del juego: identificación del ganador; orden del material, ...

La ficha resumen que se utiliza actualmente para este juego recoge este tipo de informaciones (ver figura 2):

Figura 2

Nombre del alumno: Fecha del juego

Nombre del juego:

Identificación de los dibujos del tablero en su casilla numerada correspondiente:

Número total de dibujos:

- Casillas que se va recorriendo sumando el resultado obtenido en los dados.

- Completar la serie numérica entre dos números dados, fijándose en el tablero de juego. (ej.: anotar por las casillas que pasarán si están en la 19 y te sale en los dados 24).

- Recordatorio de algunas instrucciones de avanzar o retroceder, haciéndolo por escrito en la hoja resumen, anotando las casillas por las que se pasa.

- Multiplicaciones que pueden salir a partir de los dados.

- Clasificación por orden de llegada de los nombres de los seis alumnos que han jugado mediante la numeración ordinal.

\section{EL APRENDIZAJE DEL CALCULO MEDIANTE LOS JUEGOS EN PEQUEÑO GRUPO.}

Los aspectos que podemos señalar como más positivos en la organización del aprendizaje del cálculo mediante los talleres de juego los podemos resumir en los siguientes:

- los alumnos realizan un aprendizaje funcional de la numeración y de los diversos conceptos y procedimientos del cáculo: utilizan el número y las operaciones matemáticas para resolver una «situación-problema» que tienen interés en resolver.

- en este tipo de situaciones de aprendizaje se parte y se respetan los conocimientos de matemática informal (Baroody, 1988) que los alumnos tienen en el 
momento de empezar la escuela, y se puede hacer un paso progresivo a la matemática formal que es la que se aprende en la escuela y que es necesaria para el aprendizaje posterior de conceptos matemáticos.

- el maestro puede llevar a cabo una observación de las estrategias, «errores», dificultades, procedimientos utilizados por los diferentes alumnos y hacer intervenciones que sean claramente una ayuda educativa para ellos, ya que se sitúa justamente en la franja adecuada de ayuda que el maestro debe dar a sus alumnos para ayudarles a construir los conomientos (Coll, Solé, 1989).

- la formación de grupos de alumnos en este tipo de tarea es muy beneficioso, ya que cada uno de ellos utiliza las estrategias que ha podido llegar a construir. Los compañeros aprenden unos de otros mediante la confrontación de diversos procedimientos, de diferentes experiencias extraescolares, de diversos niveles de capacidad. Así pues, consideramos que mediante este tipo de organización de clase permitimos la existencia de diversidad en los grupos y existe la posibilidad de construcción a diferentes ritmos de los conceptos matemáticos que están implicados en los juegos.

- los alumnos consiguen una agilidad importante en el cálculo mental utilizando diversas estrategias de descomposición de los números, y al mismo tiempo tienen la posibilidad de iniciarse en las primeras formalizaciones del lenguaje matemático mediante la utilización de signos representativos por escrito.

\section{Notas}

1 Este juego, así como otros de los utilizados en la clase, está extraído de la obra de C. Kamii (1986), citado en la bibliografía.

\section{Referencias}

Bassedas, E.; Eguia, E.; Gimenez, M. J.; Marced, M.; Puig, N.; Silva, M. A.; (1990). Juguem, comptem. Un taller de matemàtiques per a nens $i$ nenes de 4 a 8 anys. Barcelona: A.A.D.S.A. Rosa Sensat.

BAroody, A., (1988). El pensamiento matemático de los niños. Madrid. Visor/MEC.

Colt, C.; Sole, I., (1989). Aprendizaje significativo y ayuda pedagógica. Cuadernos de Pedagogía. $168,16-20$

KАMІІ, C.K., (1986). El niño reinventa la aritmética. Madrid. Visor.

M.E.C., (1989). Diseño Curricular Base. Educación Primaria. Madrid. M.E.C. 


\section{Utilizar el cálculo en la escuela: la programación de una situación significativa. Eulàlia Bassedas CL\&E, 1991, 11-12, pp. 87-94}

Resumen: El artículo se refiere a una experiencia sabre aprendizaje del cálculo realizada con niños de 4 a 8 años (parvulario y Ciclo inicial de Primaria). Se explicitan los aspectos básicos de su organización (utilización de juegos de mesa, trabajo en pequeño grupo, importancia de la observación sistemática), asi como las bases teóricas que la sustentan (construcción del conocimiento mediante tareas significativas, aprovechamiento de los conocimientos informales de los alumnos, importancia de la interacción entre maestra y alumnos, atención a la diversidad). Se acompañan algunos ejemplos del material elaborado (pautas de observación, ficbas-resumen).

Datos sobre la autora: Eulàlia Bassedas i Ballús trabaja en la Escola de Mestres San Cugat, de la Universidad Autónoma de Barcelona.

Dirección: Universidad Autónoma de Barcelona. Escola de Mestres Sant Cugat.

Agradecimientos: Este trabajo no habría sido posible sin la participación de las maestras Elena d'Eguia, M. José Giménez, Marta Marced, Núria Puig y M. Angels Silva, del C.P. Antonio Gaudí de Sant Boi de Llobregat (Barcelona).

(C) De todos los artículos. Deberá solicitarse por escrito autorización de CL\&E y de los autores para el uso en forma de facsímil, fotocopia o cualquier otro medio de reproducción impresa. CL\&E se reserva el derecho de interponer las acciones legales necesarias en aquellos casos en que se contravenga la ley de derechos de autor. 